\title{
SOLVABILITY OF NON-INVARIANT DIFFERENTIAL OPERATORS ON HOMOGENEOUS SPACES
}

\author{
RONALD L. LIPSMAN
}

(Communicated by Jonathan M. Rosenberg)

\begin{abstract}
We consider the solvability of non-invariant differential operators on homogeneous spaces. Such operators cannot be expected to have solutions in smooth functions (an illustrative example is provided). However, Lion has shown that, under suitable growth conditions on the infinitesimal components of the operators in a representation-theoretic decomposition, one can deduce solvability in a space of distributions. In this paper we prove that Lion's result can be improved to yield solvability in square-integrable functions.
\end{abstract}

\section{AN IMPROVEMENT OF LION'S RESULT}

Let $G$ be a connected Lie group, $H$ a closed subgroup, $\nu$ a unitary representation of $H$ and $\tau=\operatorname{Ind}_{H}^{G} \nu$. Denote by $\mathfrak{U}(\mathfrak{g})$ the (complexified) universal enveloping algebra of the Lie algebra $\mathfrak{g}$ of $G$. The elements of $\mathfrak{U}(\mathfrak{g})$ act on smooth sections in the space $\mathscr{H}_{\tau}$ of $\tau$ by differential operators. The action is as follows. Let $\delta_{H, G}$ denote the quotient of the modular functions of $H$ and $G$. Let $\mu_{G, H}$ denote a relatively invariant measure on $G / H$. Consider

$$
C^{\infty}(\tau)=\left\{f \in C^{\infty}\left(G, \mathscr{H}_{\tau}\right): f(g h)=\delta_{H, G}^{1 / 2}(h) \nu(h)^{-1} f(g), \quad h \in H, g \in G\right\} .
$$

The space $\mathscr{H}_{\tau}^{\infty}$ of $C^{\infty}$-vectors of $\tau$ lies inside $C^{\infty}(\tau)$. The action of $G$ on $L^{2}\left(G, \mathscr{H}_{\nu}, d \mu_{G, H}\right)$ by left translation is unitary, differentiates to $\mathfrak{g}$ and extends canonically to $\mathfrak{U}(\mathfrak{g})$. The resulting action of $\mathfrak{U}(\mathfrak{g})$ on $C^{\infty}(\tau)$ gives the desired differential operators. For $L \in \mathfrak{U}(\mathfrak{g})$, we denote the corresponding differential operator on $C^{\infty}(\tau)$ by $L_{\tau}$ or $\tau(L)$; and we denote the totality of them by $\mathfrak{U}_{\tau}$. We write $\mathfrak{U}_{\tau}^{0}$ for those that commute with the action of $G$, and refer to them as invariant. Typically, one has nice solvability results on the operators in $\mathfrak{U}_{\tau}^{0}$. For example, if $G / H$ is nilpotent and symmetric, and $\nu=1$, we have global solvability [1]; or if $G=G_{1} \times G_{1}, H=\Delta_{G_{1}}$ the diagonal ( $\nu=1$ again), then $\mathfrak{U}_{\tau}^{0}$ equals $\mathfrak{Z}\left(\mathfrak{g}_{1}\right)$, the center of $\mathfrak{U}\left(g_{1}\right)$, and one has local solvability [3]. The main thrust of the subject (see [2], [7]) is to exploit the solvability of the operators

Received by the editors November 17, 1988 and, in revised form, January 30, 1989.

1980 Mathematics Subject Classification (1985 Revision). Primary 22E99; Secondary 58G99.

This research was supported by NSF under MCS 87-00551A01. 
in $\mathfrak{U}_{\tau}^{0}$ and a Plancherel decomposition of $\tau$ to obtain solvability criteria for the operators in $\mathfrak{U}_{\tau}$. Helffer-Nourrigat [5] were the first to extend that thrust from groups to homogeneous spaces. Lion [6] has given a quite general result on local solvability in a space of distributions for these operators. Our main theorem is that Lion's result can be improved to give $L^{2}$-solvability. We also give an example which shows that solvability in $C^{\infty}$ is often false.

We simplify the presentation by assuming $\nu=1$. The resulting induced representation $\tau=\operatorname{Ind}_{H}^{G} 1$ is the quasi-regular representation of $G$ on

$$
L^{2}\left(G / H, d \mu_{G, H}\right) \text {. }
$$

Instead of $C^{\infty}(\tau)$ we write

$$
C^{\infty}(G, H)=\left\{f \in C^{\infty}(G): f(g h)=\delta_{H, G}^{1 / 2}(h) f(g), \quad h \in H, g \in G\right\} .
$$

The algebra $\mathfrak{U}_{\tau}$ is now the algebra of differential operators on $C^{\infty}(G, H)$ generated by $\tau(X), X \in \mathfrak{g}$. The subalgebra $\mathfrak{U}_{\tau}^{0}$ constitutes those that are left $G$-invariant.

For any unitary representation $\pi$ of $G$ in a separable Hilbert space $\mathscr{H}_{\pi}$, the Sobolev spaces

$$
\begin{aligned}
\mathscr{H}_{\pi}^{\infty} & \subset \cdots \subset \mathscr{H}_{\pi}^{k+1} \subset \mathscr{H}_{\pi}^{k} \subset \cdots \subset \mathscr{H}_{\pi} \\
& =\mathscr{H}_{\pi}^{0} \subset \cdots \subset \mathscr{H}^{-k} \subset \mathscr{H}^{-(k+1)} \subset \cdots \subset \mathscr{H}_{\pi}^{-\infty}, \quad k \geq 0,
\end{aligned}
$$

are described in [4] or [9]. If we apply those constructions to $\tau$, we obtain the Sobolev spaces $C^{k}(G, H)$ of $k$-times continuously differentiable functions. We write $C_{c}^{k}(G, H)$ for the subspace of functions which are compactly supported $\bmod H$. We denote their duals by $\mathfrak{H}^{-k}(G, H), k \geq 0$. Then the inductive limit $\mathfrak{H}^{-\infty}(G, H)$ is the space of distributions corresponding to the quasi-regular representation $\tau$. (For all this see [6].)

The notions of solvability for operators $L \in \mathfrak{U}_{\tau}$ considered by Lion are:

Local Solvability $-\forall f \in C^{\infty}(G, H), \forall x \in G, \exists \mathscr{U}$ neighborhood of $x$ and $u \in C^{\infty}(G, H) \ni L u=f$ in $\mathscr{U}$;

Semiglobal Solvability- $\forall f \in C_{c}^{\infty}(G, H), \exists u \in C^{\infty}(G, H) \ni L u=f$ in Supp $f$;

Global Solvability- $\forall f \in C^{\infty}(G, H), \exists u \in C^{\infty}(G, H) \ni L u=f$;

Solvability in Distributions- $\forall f \in C_{c}^{\infty}(G, H), \exists u \in \mathfrak{H}^{-\infty}(G, H) \ni L u=f$ in $\operatorname{Supp} f$.

Now I will paraphrase Lion's main theorem.

Theorem 1. Suppose $\tau$ is type $\mathrm{I}$ and $\tau=\int_{S}^{\oplus} \mathscr{H}_{\pi} d \mu(\pi)$ is a direct integral decomposition of $\tau$ into irreducibles. Let $L \in \mathfrak{U}_{\tau}$ and $Z_{1}, Z_{2} \in \mathfrak{U}_{\tau}^{0}$. Suppose that $Z_{1}$ is semiglobally solvable; and suppose that for $\mu$ a.e. $\pi$, the operator $\pi(L)$, considered as an operator on $\mathscr{H}_{\pi}^{\infty}$, has a right inverse $\pi(L)^{-1}$. For $k, l \in \mathbf{Z}$ 
write $\left\|\pi(L)^{-1}\right\|_{k, l}$ to denote the norm of $\pi(L)^{-1}$ considered as an operator from $\mathscr{H}_{\pi}^{k}$ to $\mathscr{H}_{\pi}^{l}$. Suppose that

$$
\left|\pi\left(Z_{1}\right)\right||| \pi(L)^{-1} \|_{k, l} \leq\left|\pi\left(Z_{2}\right)\right|
$$

is true $\mu$ a.e. either

(i) for all $k, l \in \mathbf{Z}$, or

(ii) for some $k, l \in \mathbf{Z}$.

Then in case (i) we conclude that $L$ is semiglobally solvable; whereas in case (ii) we conclude that $L$ is solvable in distributions.

Remarks. (1) The operators $\pi\left(Z_{j}\right), j=1,2$, are scalar since the $Z_{j}$ must be diagonalizable for the direct integral decomposition.

(2) An examination of Lion's proof [6, p. 252ff] reveals that the precise conclusion in case (ii) is that the equation

$$
L u=f \in C_{c}^{\infty}(G, H)
$$

is solvable for $u \in \mathfrak{H}^{-\operatorname{deg} Z_{2}}(G, H)$.

(3) A condition like (i) is very difficult to substantiate. In fact, typically, the best we can hope for is condition (ii) - usually (although not always) with $k=l=0$. That is certainly what happens in much of the prior work in the subject (see [2], [7]). In that case Lion's theorem only gives solvability in distributions. The main goal of this paper is to show that actually $L^{2}$-solvability is attainable. We make that precise in our main

Theorem 2. Let the hypotheses be as in Theorem 1. Assume condition (ii) is true with $k=l=0$. Then the equation

$$
L u=f \in C_{c}^{\infty}(G, H)
$$

can be solved with $u \in L^{2}\left(G, H, d \mu_{G, H}\right)$.

Proof. Let

$$
\Phi: L^{2}\left(G, H, d \mu_{G, H}\right) \rightarrow \int_{S}^{\oplus} \mathscr{H}_{\pi} d \mu(\pi)
$$

be the intertwining operator that decomposes $\tau$ into $\int_{S}^{\oplus} \pi d \mu(\pi)$. If we denote the $\pi$-th component of $\Phi f$ by $\Phi_{\pi} f$, then $\Phi$ satisfies

$$
\Phi_{\pi} \tau(g) f=\pi(g) \Phi_{\pi} f, \quad f \in L^{2}\left(G, H, d \mu_{G, H}\right), g \in G .
$$

Differentiating and extending the representation gives us the equations

$$
\begin{array}{ll}
\Phi_{\pi} \tau(X) f=\pi(X) \Phi_{\pi} f, & X \in \mathfrak{g}, \\
\Phi_{\pi} \tau(L) f=\pi(L) \Phi_{\pi} f, & L \in \mathfrak{U}(\mathfrak{g}) .
\end{array}
$$

A fundamental fact, due to Goodman [4], says that for $k \geq 0$ we have

$$
f \in \mathscr{H}_{\tau}^{k}=C^{k}(G, H) \quad \Leftrightarrow \quad \Phi_{\pi} f \in \mathscr{H}_{\pi}^{k} \text { for } \mu \text { a.a. } \pi \text {. }
$$


Now let $f_{1} \in C_{c}^{\infty}(G, H), L \in \mathfrak{U}(\mathfrak{g})$, and consider the equation

$$
L u=f_{1} ;
$$

which, of course, is just shorthand notation for

$$
\tau(L) u=f_{1} .
$$

We define a conjugate-linear functional $\Omega$ in $C_{c}^{\infty}(G, H)$ as follows:

$$
\Omega: \varphi \rightarrow \int\left\langle\pi(L)^{-1} \Phi_{\pi} f_{1}, \Phi_{\pi} \varphi\right\rangle \pi\left(Z_{1}\right) d \mu(\pi), \quad \varphi \in C_{c}^{\infty}(G, H) .
$$

According to the hypothesis of the theorem the integrand is finite-valued for $\mu$ a.e. $\pi$. But much more is true. We have

$$
\begin{aligned}
|\Omega(\varphi)| & =\left|\int\left\langle\pi(L)^{-1} \Phi_{\pi} f_{1}, \Phi_{\pi} \varphi\right\rangle \pi\left(Z_{1}\right) d \mu(\pi)\right| \\
& \leq \int\left|\left\langle\pi(L)^{-1} \Phi_{\pi} f_{1}, \Phi_{\pi} \varphi\right\rangle\right|\left|\pi\left(Z_{1}\right)\right| d \mu(\pi) \\
& \leq \int\left|\pi\left(Z_{1}\right)\right|\left\|\pi(L)^{-1}\right\|\left\|\Phi_{\pi} f_{1}\right\|\left\|\Phi_{\pi} \varphi\right\| d \mu(\pi) \\
& \leq \int\left|\pi\left(Z_{2}\right)\right|\left\|\Phi_{\pi} f_{1}\right\|\left\|\Phi_{\pi} \varphi\right\| d \mu(\pi) \\
& =\int\left\|\Phi_{\pi}\left(\tau\left(Z_{2}\right) f_{1}\right)\right\|\left\|\Phi_{\pi} \varphi\right\| d \mu(\pi) \\
& \leq\left(\int\left\|\Phi_{\pi}\left(\tau\left(Z_{2}\right) f_{1}\right)\right\|^{2} d \mu(\pi)\right)^{1 / 2}\left(\int\left\|\Phi_{\pi} \varphi\right\|^{2} d \mu(\pi)\right)^{1 / 2} \\
& =\left\|\tau\left(Z_{2}\right) f\right\|_{2}\|\varphi\|_{2} .
\end{aligned}
$$

That is the conjugate-linear functional $\Omega$ is bounded on $L^{2}$. Hence there exists $u \in L^{2}\left(G, H, d \mu_{G, H}\right)$ such that

$$
\Omega(\varphi)=\langle u, \varphi\rangle, \quad \forall \varphi \in C_{c}^{\infty}(G, H) .
$$

But for an arbitrary test function $\psi \in C_{c}^{\infty}(G, H)$, we have

$$
\begin{aligned}
\langle\tau(L), u, \psi\rangle & =\left\langle u, \tau(L)^{*} \psi\right\rangle=\Omega\left(\tau(L)^{*} \psi\right) \\
& =\int\left\langle\pi(L)^{-1} \Phi_{\pi} f_{1}, \Phi_{\pi} \tau(L)^{*} \varphi\right\rangle \pi\left(Z_{1}\right) d \mu(\pi) \\
& =\int\left\langle\pi(L)^{-1} \Phi_{\pi} f_{1}, \pi(L)^{*} \Phi_{\pi} \varphi\right\rangle \pi\left(Z_{1}\right) d \mu(\pi) \\
& =\int\left\langle\Phi_{\pi} f_{1}, \Phi_{\pi} \psi\right\rangle \pi\left(Z_{1}\right) d \mu(\pi) \\
& =\left\langle\tau\left(Z_{1}\right) f_{1}, \psi\right\rangle .
\end{aligned}
$$

Therefore

$$
\tau(L) u=\tau\left(Z_{1}\right) f_{1}
$$


Finally, let $f \in C_{c}^{\infty}(G, H)$. By the hypothesis of semiglobal solvability of $Z_{1}$, there exists $v \in C^{\infty}(G, H)$ such that

$$
\tau\left(Z_{1}\right) v=f \quad \text { on } \operatorname{Sup} f .
$$

Now choose $w \in C^{\infty}(G)$ satisfying: $w(g h)=w(g), g \in G, h \in H ; w \equiv 1$ on $\operatorname{Sup} f \cdot H$; and $w$ is compactly supported $\bmod H$. Then $f_{1}=v w \in$ $C_{c}^{\infty}(G, H)$ and we have

$$
\tau\left(Z_{1}\right) f_{1}=f \quad \text { on } \operatorname{Sup} f .
$$

Combining with equation $(\mathrm{A})$, we obtain $\tau(L) u=\tau\left(Z_{1}\right) f_{1}=f \quad$ on $\operatorname{Sup} f$.

Examples. In this section we give examples to illustrate several themes relevant to Theorems 1 and 2. The examples are based on the work in [7].

(a) Suppose $\mathfrak{g}$ has three generators $T, X, Y$ satisfying bracket relations

$$
[T, X]=Y, \quad[T, Y]=X .
$$

We take $G$ to be the corresponding simply connected exponential solvable Lie group and $H=\exp \mathbf{R} T$. The homogeneous space $G / H$ is an abelian symmetric space. By [8]

$$
\tau=\operatorname{Ind}_{H}^{G} 1 \cong \int_{\widehat{N} / H}^{\oplus} \pi_{\chi} d \dot{\chi},
$$

where $\pi_{\chi}=\operatorname{Ind}_{N}^{G} \chi, \chi \in \widehat{N}, N=\exp (\mathbf{R} X+\mathbf{R} Y), d \dot{\chi}=$ a push-forward of Lebesgue measure from $\widehat{N}$ to $\widehat{N} / H$. As in [7] we consider

$$
L=L_{\lambda}=A^{2}+X^{2}+\lambda Y, \quad \lambda \in \mathbf{R} .
$$

But unlike [7] we consider $L$ as a non-invariant operator on $G / H$. Now the spectra of $\tau$ and of the regular representation are the same (the multiplicities are different). Therefore, examining [7, $\S 5$, particularly p. 1311], we see that the conditions of Theorem 2 are satisfied with $\operatorname{deg} Z_{2}=1$. Thus Lion's result gives solvability of $L$ in $\mathfrak{H}^{-1}(G, H)$; but Theorem 2 yields solvability in $L^{2}$. If we choose coordinates $(x, y) \leftrightarrow \exp (x X+y Y)$ on $G / H \approx N$, the operator becomes

$$
L=\left(x \frac{\partial}{\partial y}+y \frac{\partial}{\partial x}\right)^{2}+\frac{\partial^{2}}{\partial x^{2}}+\lambda \frac{\partial}{\partial y} .
$$

I do not know whether this operator has smooth solutions.

(b) Let $G=H N$ be a metabelian semidirect product, $H=\exp \mathbf{R} T \cong \mathbf{R}, N$ a normal vector group. Suppose the Lie algebra $\mathfrak{n}$ of $N$ is spanned by $X$ and $Y_{j}, 1 \leq j \leq n(n>1)$; and suppose the bracket relations are

$$
\begin{aligned}
{[T, X] } & =X, \\
{\left[T, Y_{j}\right] } & =\rho_{j} Y_{j}, \quad \rho_{j} \text { real } .
\end{aligned}
$$


Assume there exist nonnegative integers $n_{j}$ such that

$$
\begin{gathered}
\sum n_{j} \rho_{j}=0, \\
N=\sum n_{j}>1 \quad(\text { see [7]). }
\end{gathered}
$$

Consider the element $L$ of $\mathfrak{U}(\mathfrak{g})$ given by

$$
L=T-\sum_{j=1}^{n} Y_{j}^{2}
$$

viewed as an operator on $G / H$. Once again $G / H$ is an abelian symmetric space, so $\tau$ and the regular representation of $G$ have the same spectrum. An examination of the analysis in [7, $\S 4$, particularly p. 1295] reveals that, once again, both Theorems 1 and 2 apply with $\operatorname{deg} Z_{2}=1$. Thus Theorem 2 gives $L^{2}$-solvability of $L$. But this time we can be assured-by the term $x \partial / \partial x$ in the expression of $L$ in the coordinates $\left(x, y_{j}\right) \leftrightarrow \exp x X \exp \left(\sum y_{j} Y_{j}\right)$-that $L$ is not solvable in smooth functions. We leave it to the reader to jack up the example so that $\operatorname{deg} Z_{2}$ is as high as one desires.

\section{REFERENCES}

1. Y. Benoist, Analyse harmonique sur les espaces symétriques nilpotents, J. Funct. Anal. 59 (1984), 211-253.

2. L. Corwin, A representation theoretic criterion for local solvability of left-invariant differential operators on nilpotent Lie groups, Trans. Amer. Math. Soc. 264 (1981), 113-120.

3. M. Duflo, Opérateurs différentiels bi-invariants sur un group de Lie, Ann. Scient. Ec. Norm. Sup. 10 (1977), 265-288.

4. R. Goodman, One-parameter groups generated by operators in an eveloping algebra, J. Funct. Anal. 6 (1970), 218-236.

5. B. Helffer and J. Nourrigat, Hypollepticité pour des groups nilpotents de rang 3, Comm. Part. Diff. Eqns. 3 (1978), 643-743; see also CPDE 4 (1979), 899-958.

6. G. Lion, Résolubilité d'équations différentielles et représentations induites, J. Funct. Anal. $\mathbf{7 5}$ (1987), 211-259.

7. R. Lipsman, Solvability of invariant differential operators with variable coefficients, Comm. Part. Diff. Eqns. 10 (1985), 1261-1316.

8. __, Harmonic analysis on non-semisimple symmetric spaces, Israel J. Math. 54 (1986), 335350.

9. __ Sobolev criteria for solvability of invariant differential operators, Comm. Part. Diff. Eqns. 12 (1987), 327-349.

Department of Mathematics, University of Maryland, College Park, Maryland 20742 